1

2

3

4

5

$6 \quad{ }^{1}$ National Key Laboratory of Plant Molecular Genetics, CAS Center for Excellence in Molecular

7 Plant Sciences, Shanghai Institute of Plant Physiology and Ecology, Chinese Academy of Sciences,

8 Shanghai 200032, China

9

10

\section{Asymmetric Redundancy of Soybean Nodule Inception (NIN) Genes in Root Nodule Symbiosis}

\footnotetext{
${ }^{2}$ University of the Chinese Academy of Sciences, Beijing, China
}

${ }^{3}$ College of Resources and Environment, Fujian Agriculture and Forestry University, Fuzhou, Fujian, 350002, China

${ }^{4}$ FAFU-UCR Joint Center for Horticultural Biology and Metabolomics, Fujian Agriculture and Forestry University, Fuzhou, 350002, China

* To whom correspondence should be addressed: fxie@cemps.ac.cn; guan@fafu.edu.cn

$1 \mathrm{M} . \mathrm{Fu}, \mathrm{X}$. Li and F. Xie designed the research, M. Fu and X. Li conducted experiments and analyzed data. J. Sun and Y. Guan generated and scored the

3 CRISPR-Cas9 mutants. F. Xie and M. Fu wrote the paper. All authors read and 
24

25

26

27

28

29

30

31

32

33

34

35

36

37

approved the final article.

5

\section{ABSTRACT}

NIN is one of the most important root nodule symbiotic genes as it is required for both infection and nodule organogenesis in legume. Unlike most legumes with a sole NIN gene, there are four putative NIN genes in soybean (Glycine max). Whether and how these orthologs NIN genes contribute to soybean-rhizobia symbiotic interaction remain unknown. In this study, we found that all four GmNIN genes are induced by rhizobia, and that conserved $\mathrm{CE}$ and $\mathrm{CYC}$ binding motifs in their promoter regions are required for their expression in the nodule formation process. By generation of multiplex Gmnin mutants, we found that Gmnin 1a nin2a nin2b triple mutant and Gmnin1a nin1b nin2a nin2b quadruple mutant displayed similar defects in rhizobia infection and root nodule formation, Gmnin $2 a \operatorname{nin} 2 b$ produced less nodules but displayed hyper infection phenotype than wild type, while a Gmnin 1a nin $1 b$ double mutant nodulated as wild type. Overexpression of GmNIN1a, GmNIN1b, GmNIN2a, and GmNIN2b reduced nodule numbers after rhizobia inoculation, with GmNIN1b overexpression having the weakest effect. In addition, overexpression of GmNIN1a, GmNIN2a, or GmNIN2b, but not GmNIN1b, produced malformed pseudo-nodule like structures without rhizobia inoculation. In conclusion, GmNIN1a, GmNIN2a and

GmNIN2b play functionally redundant yet complicated roles for soybean nodulation. 
50 GmNIN1b, although is expressed at comparable level with other homologs, plays a minor role in root nodule symbiosis. Our work provides insight into the understanding of asymmetrically redundant function of GmNIN genes in soybean.

\section{INTRODUCTION}

The legume-rhizobia symbiosis is the most important symbiotic association in terms of biological nitrogen fixation. In root nodules, endosymbiotic rhizobia use carbon from their legume hosts to fix atmospheric nitrogen into ammonia to provide the host plant with nitrogen nutrition. Understanding the underlying mechanisms of nodulation and nitrogen fixation is very important for improving agricultural production and for insights into natural ecosystems.

The legume-rhizobia symbiotic interaction is initiated by mutual recognition of molecular signals. The process is initiated by plant roots secreting flavonoid compounds into the soil, this attracts compatible rhizobia and stimulates them to synthesize and secrete highly specific lipochito-oligosaccharide (LCO) signalling molecules called nodulation factors (NFs) (Liu et al., 2016). Legume plants perceive NF signals via LysM receptor-like kinase expressed in their roots. NF recognition triggers two coordinated plant developmental programs: initiation of the infection process by which the bacteria enter the host cells, and, simultaneously, the elicitation of pericycle and cortical cell division resulting in nodule organ formation (Murray, 2011). When the bacteria reach the nodule primordium cells through the infection threads they are released into host-membrane enclosed structures called symbiosomes where they fix nitrogen (Oldroyd et al., 2011).

Soybean (Glycine max) is one of the most important legume crops in the world, and provides human food, animal feed, nutritional by-products and vegetable oil for human consumption and biofuel production. As a legume, soybean also has the capability to form root nodules through association with Rhizobium bacteria. The 
77 soybean genome was sequenced (Schmutz et al., 2010) and transcriptome and 78 proteome analyses after rhizobia or NF inoculation have been performed in a number 79 of studies (Hayashi et al., 2012; Libault et al., 2010; Libault et al., 2009; etc.). Understanding the biological function of the genes involved will help to establish elite cultivars that benefit sustainable farming practices. Forward and reverse genetic methods are available for soybean. However, due to multiple genome duplications that occurred at approximately 59 and 13 million years ago, approximately $75 \%$ of soybean genes are present in multiple copies leading to high genetic redundancy (Schmutz et al., 2010). Duplication occurred in segmental regions, accompanied by local genomic as well as sequence divergences. For example, the Nod factor receptor genes NFR1 and NFR5 are duplicated in soybean (GmNFR1 $\alpha-$ GmNFR1 $\beta$ and GmNFR5 $\alpha-$ GmNFR5 $\beta$ ). GmNFR1 $\alpha$ and GmNFR1 $\beta$ have $92 \%$ identity at the nucleotide level, but have different functions (Indrasumunar et al., 2010). On the other hand, GmNFR5 $\alpha$ and GmNFR5 $\beta$ have 95\% nucleotide identity and can functionally complement each other (Indrasumunar et al., 2011). The Symbiosis Receptor Kinase $(\operatorname{SymRK})$ gene also has two copies in soybean $(\mathrm{GmSymRK} \alpha$ and GmSymRK $\beta)$. RNAi knock down of the GmSYMRK genes revealed that GmSymRK $\beta$ is more important for root endosymbiosis than $G m S y m R K \alpha$, suggesting it retained its function after duplication (Indrasummunar et al., 2015).

NIN (Nodule Inception) is one of the most important genes in nodulation and is the founder gene for the NIN-like protein (NLP) transcription factor family in plants, which contain a conserved RWP-RK DNA-binding domain and a Phox and Bem1 (PB1) dimerization domain (Griesmann et al., 2018; Schauser et al., 1999; van Velzen et al., 2018). NIN has been shown to be required for rhizobial infection and nodule organogenesis in several legumes, including M. truncatula, L. japonicus and Pisum sativum (Marsh et al., 2007; Schauser et al., 1999; Borisov et al., 2003). LjNIN can directly bind to promoters of infection-specific genes and activate their expression, such as NPL (Nodulation pectate lyase), SCARN (SCAR Nodulation) and EPR3 (EPS receptor 3) (Xie et al., 2012; Qiu et al., 2015; Kawaharada et al., 2017). NIN's targets 
106 include genes which function in cortical cell division for root nodule organogenesis in 107 L. japonicus and M. truncatula, including two Nuclear Factor-Y (NF-Y) subunit genes 108 (LjNF-YA1 and LjNF-YB1), the cytokinin receptor MtCRE1, and LjLBD16a/ASL18 109 (Soyano et al., 2013; Vernie et al., 2015; Soyano et al., 2019). In addition, NIN has an 110 essential role in the autoregulation of nodulation (AON) signaling pathway through its 111 direct control of CLE-Root Signal1/2 (LjCLE-RS1/2) or MtCLE13/35 expression 112 which act to limit nodule numbers (Soyano et al., 2014; Laffont et al., 2020; Luo et al., 113 2021). NIN can interact with other NLPs, including NLP1 which has important role in 114 regulating nitrate inhibition of nodulation (Lin et al., 2018). Moreover, studies from 115 non-legume nodulating plants, including CgNIN from the actinorhizal tree Casuarina 116 glauca and PaNIN from Parasponia, showed that NIN is essential gene for 117 actinorhizal and rhizobial nodulation, respectively (Clavijo et al., 2015; Bu et al., 118 2020). Thus, NIN is important for symbiotic nodulation, including rhizobial infection, 119 nodule organogenesis, and the dynamic regulation of nodule numbers by nitrate 120 through AON. In soybean, microarray data showed that several NIN genes were 121 induced by rhizobia, but their function in nodulation has not been studied.

122 In this work, we investigated the expression patterns of the GmNIN genes, and 123 used an RNAi knock downs strategy, CRISPR-Cas9 knock out mutants, and 124 overexpression to fully analyze their function during the nodulation process. Our 125 results show that GmNIN1a, GmNIN2a, and GmNIN2b function redundantly to 126 mediate rhizobia infection and nodule initiation, and that GmNIN1b plays only a 127 minor role. 


\section{RESULTS}

131 GmNINs are Induced by Rhizobia and Have Nodulation-Specific Expression Patterns

133 The G. max genome encodes four GmNIN proteins belonging to the same clade as 134 LjNIN and MtNIN (Lin et al., 2018) (Supplemental Figure S1). We then analyzed the 135 gene expression patterns of the GmNINs by RT-qPCR after rhizobia inoculation. Our 136 results showed that all four GmNINs can be induced by rhizobia and the induction

137 levels were highest after 2 weeks post inoculation (Figure 1, A-D), and highly 138 expressed in nodules (Figure 1, E-H). This result was consistent with previous 139 microarray data that these GmNINs are highly induced during nodulation process 140 (Libault et al.,2010). 
141 The promoter of NIN has a CYC motif (for CYCLOPS binding site) which is 142 required for expression of NIN in epidermal cells to regulate rhizobial infection, and a 143 remote conserved region with putative cytokinin response elements (CE region) 144 which is required for expression of NIN in the pericycle to initiate nodule primordium 145 formation in L. japonicus and M. truncatula (Singh et al., 2014; Liu et al., 2019). 
146 Analysis of all four GmNIN promoter regions revealed CYC motifs within $3 \mathrm{~kb}$

147 upstream of their start codons (Supplemental Figure S2 and Table S1), and two

148 (GmNIN1a and GmNIN2a) or three (GmNIN1b and GmNIN2b) conserved regions far

149 upstream of their start codon (Supplemental Table S1). The $2^{\text {nd }}$ region was the most

150 conserved region, which contains several putative cytokinin response elements in the 
151 promoter of MtNIN (Liu et al., 2019). Even all the 4 GmNINs contains the $2^{\text {nd }} C E$

152 region, it was conserved in GmNIN1a, NIN2a and NIN2b, but not in GmNIN1b

153 promoter (Supplemental Figure S3). In order to further analyze the expression 154 patterns of GmNINs, we made two promoter-GUS reporter constructs, one with the 155 promoter of GmNIN2a containing its CYC motif ( $\left.p N I N 2 a_{C Y C}\right)$ and a second version 

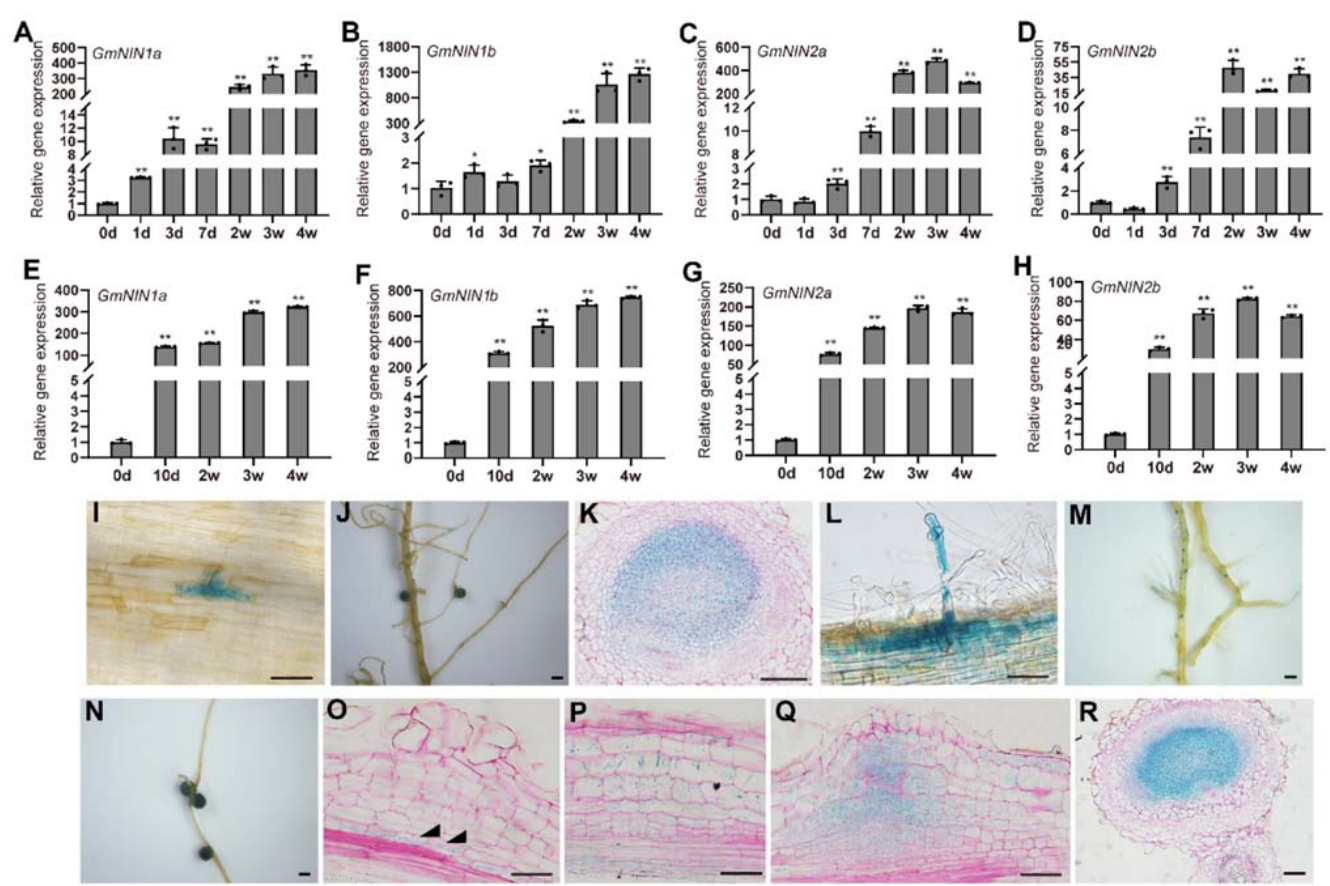

L
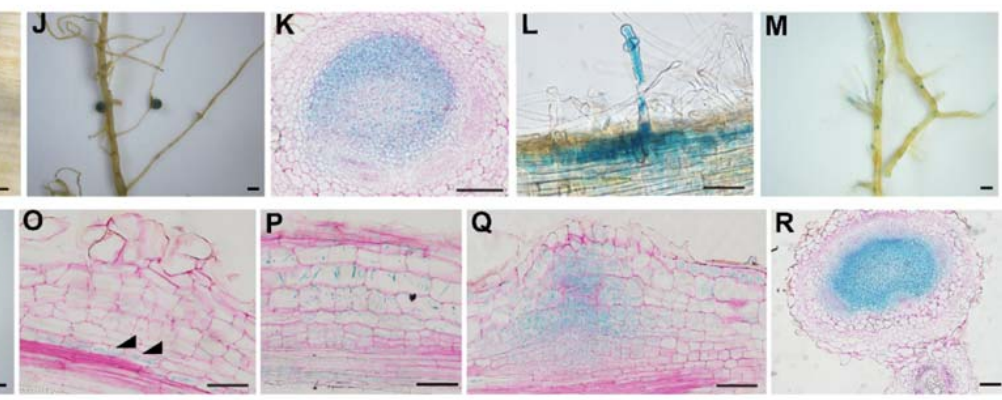

Q

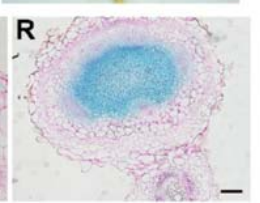

Figure 1. GmNINs were induced in soybean roots and nodules by rhizobia inoculation and have nodulation-specific expression patterns. Ato H, Expression levels of GmNIN1a (A, E), GmNIN1b (B, F), GmNIN2a (C, G) and GmNIN2b (D, H) in G. $\max$ roots (A-D) and nodules (E-H) after rhizobia inoculation. RT-qPCR were used to measure gene expression levels, normalized relative to uninoculated roots $\left(\begin{array}{ll}0 & \mathrm{~d}\end{array}\right)$. Values are means $\pm \mathrm{SE}$. Data shown are from one representative experiment of three biological replicates. Asterisks indicate significant differences between treated and control conditions ( $0 \mathrm{~d})$ (Student's $t$-test, $* P<0.05$, ** $P<0.01$ ). I to $\mathrm{R}$, The $p G m N I N 2 a_{C Y C}: G U S(\mathrm{I}-\mathrm{K}), p G m N I N 2 a_{C E-C Y C}$ GUS (L-R) were expressed in G. max hairy roots, and the images were taken at 3 days (I, L), 5 days (M) or 14 days (J, N) after rhizobia inoculation. (K, O-R) Section of these nodules stained with ruthenium red. Arrows (O) indicate GUS expression in pericycle cells. Scale bars: $1 \mathrm{~mm}(\mathrm{~J}, \mathrm{M}$ and $\mathrm{N}) ; 100 \mu \mathrm{m}(\mathrm{K}, \mathrm{Q}$ and R); $50 \mu \mathrm{m}(\mathrm{I}, \mathrm{L}$, $\mathrm{O}$ and $\mathrm{P})$.

containing the $2^{\text {nd }}$ CE region and CYC motif ( $\left.p N I N 2 a_{C E-C Y C}\right)$ fusion GUS reporter gene and expressed them in G. max by hairy root transformation. After rhizobia inoculation, weak GUS expression was detected in curled root hairs and nodules of $p N I N 2 a_{C Y C}$ :GUS transgenic roots (Figure 1, I and J). In contrast, in roots transformed with the $p N I N 2 a_{C E-C Y C}$ :GUS construct the GUS expression was much stronger, and 
161 staining was seen in root hairs, nodule primordia and nodules (Figure 1, L-N).

162 Sectioning of the nodules revealed that both $p N I N 2 a_{C Y C}$ and $p N I N 2 a_{C E-C Y C}$ expressed

163 in the nodule fixation zone, but the $p N I N 2 a_{C E-C Y C}$ expression was stronger than $164 p N I N 2 a_{C Y C}$ (Figure $1, \mathrm{~K}$ and $\left.\mathrm{R}\right)$. However, only $p N I N 2 a_{C E-C Y C}:$ GUS transgenic roots 165 showed GUS activity in divided pericycle and cortex cells (Figure 1, O-Q). We 166 further deleted $(\Delta)$ the D1, D2 or D3 in the $2^{\text {nd }} \mathrm{CE}$ region, and found that $\Delta \mathrm{D} 1$ 167 displayed normal expression pattern, but only half of the transgenic plants can detect 168 weaker GUS expression (Supplemental Figure S4, A-F and Table S2). However, $\Delta$ D2 169 or $\Delta \mathrm{D} 3$ barely affected GmNIN2a expression pattern and expression level 170 (Supplemental Figure S4, G-L, M-R and Table S2). This result was consistent with $171 M t N I N$, which showed that $p \operatorname{MtNIN}_{C E(\triangle \mathrm{D} 1)-C Y C}$ :GUS cannot rescue nin-1 nodulation 172 phenotype (Liu et al., 2019), and suggested that the D1 motif is essential for GmNINs 173 expression.

174 We then analyzed the GmNINs protein sequence, and found that the C-terminal RWP-RK and PB1 domain are conserved in the GmNINs, but the GmNIN1b N-terminal was shorter than other GmNINs (Supplemental Figure S5). Then the subcellular localization of GmNINs were examined using N-terminal GFP fusion proteins which were expressed in $N$. benthamiana leaves. Our result showed that these four GmNIN proteins displayed nuclear localization (Supplemental Figure S6).

\section{Silencing of GmNINs Using RNAi Reduced Nodule Numbers}

182

In order to investigate the role of GmNINs in the root nodule symbiosis, we first used RNA interference (Ri) to reduce their transcript levels and nodule numbers were analyzed at 21 days post inoculation (dpi) with rhizobia. We found that GmNINla Ri plants produced no nodules; GmNIN2a $R i$ and GmNIN2b Ri transgenic roots had significantly reduced nodule numbers, while GmNIN1b Ri nodule numbers were less than the EV control but more than GmNIN1a Ri, GmNIN2a Ri and GmNIN2b Ri (Figure 2, A-F). The transcript levels of the GmNINs were analyzed by RT-qPCR, and 

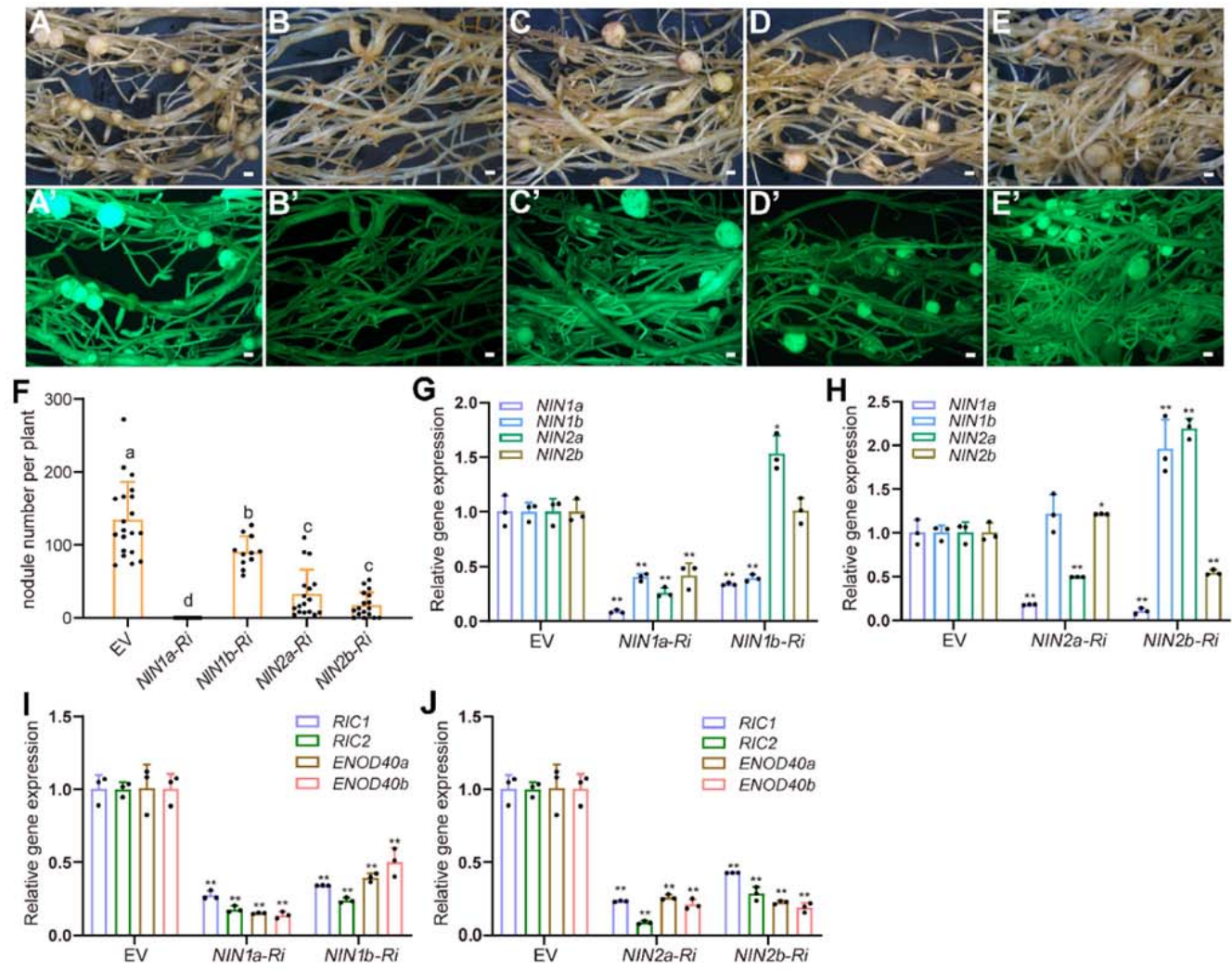

Figure 2. Nodulation phenotypes in GmNIN RNAi hairy roots. A to E, Nodule phenotypes of EV control (A), GmNINIa (B), GmNINIb (C), GmNIN2a (D) and GmNIN2b (E) RNAi transgenic roots 3 weeks after rhizobia inoculation. Upper panels (A-E) show bright-field images and the lower panels (A'-E') are epifluorescence microscopy images showing GFP expression in the same transgenic roots. Scale bars $=1 \mathrm{~mm}$. F, Nodule numbers of the EV and RNAi transgenic lines. The nodule numbers were scored at 3 weeks post inoculation with B. japonicum USDA110. Data from one representative experiment of three biological replicates. Error bars indicate SE, dots represent individual nodule numbers. The different letters indicate significant differences (one-way ANOVA-multiple comparisons). G to J, RT-qPCR analyze of the relative expression levels of GmNINs (G and $\mathrm{H}$ ) and symbiotic reporter genes (I and J) in EV and GmNINs RNAi transgenic roots at 7 days post inoculation with B. japonicum USDA110. Error bars indicate SE. Data shown are from one representative experiment using three biological replicates. Asterisks indicate significant differences $\left({ }^{*} P<0.05,{ }^{* *} P<0.01\right.$, Student's $t$-test, comparisons between EV control and experimental groups).

the results showed that GmNINla Ri reduced expression of all four GmNINs, while GmNIN1b Ri, GmNIN2a Ri and GmNIN2b Ri reduced expression of just two of the four GmNINs (Figure 2, G-H). We then analyzed GmRIC1, GmRIC2 and GmENOD40 genes expression levels in the GmNINs Ri roots. The RT-qPCR results showed that the expression of these genes was strongly reduced in the GmNIN Ri lines (Figure 2, I-J). 
194 These results suggest that these four GmNINs act redundantly in root nodule 195 formation.

GmNINs CRISPR-Cas9 Mutants were Deficient in Rhizobia Infection and

\section{Nodule Organogenesis}

199 To verify the function of GmNINs in the root nodule symbiosis process, we used multiplex mutagenesis via pooled CRISPR-Cas9 to generate high order Gmnin 201 mutants (Bai et al., 2020). Vectors with small guide RNAs (sgRNAs) targeting the conserved RWP-RK motif or the N-terminus of GmNIN1a/1b or GmNIN2a/2b were constructed, respectively. These vectors were pooled transformed into soybean cultivar, Huachun 6, using Agrobacterium tumefaciens-mediated transformation. In T2 progenies, Gmnin1b, Gmnin 1a nin1b and Gmnin2a nin2b double, Gmnin1a nin2a $\operatorname{nin} 2 b$ triple and Gmnin1a nin1b $\operatorname{nin} 2 a \operatorname{nin} 2 b$ quadruple mutants were successfully obtained. These contained insertion or deletions that led to frameshifts which generated premature stop codons (Supplemental Figure S7).

The nodulation phenotypes of these mutants were further analyzed by inoculation with $B$. japonicum USDA110 which constitutively expresses the GUS reporter gene. Gmnin $1 b$ and Gmninla nin $1 b$ produced a similar number of nodules as wild type, Gmnin2a nin2b produced less nodule numbers than wild type, and

213 Gmnin1a nin2a nin2b and Gmnin1a nin1b nin2a nin2b mutants did not develop any 214 nodules at 21 dpi (Figure 3, A and B). RT-qPCR analyze revealed that RIC1, RIC2 and 215 ENOD40 expression were reduced in these mutants, and the expression levels were 216 much lower in the Gmnin1a nin2a nin2b and Gmnin1a nin1b nin2a nin2b mutants 217 (Figure 3, C-E).

218 We further analyzed the root hair deformation and rhizobial infection thread 219 formation phenotypes of these Gmnin mutants. In wild type and Gmnin la nin $1 b$, the 220 root hairs were deformed and curled (Figure 4, A and B), and similar infection events 

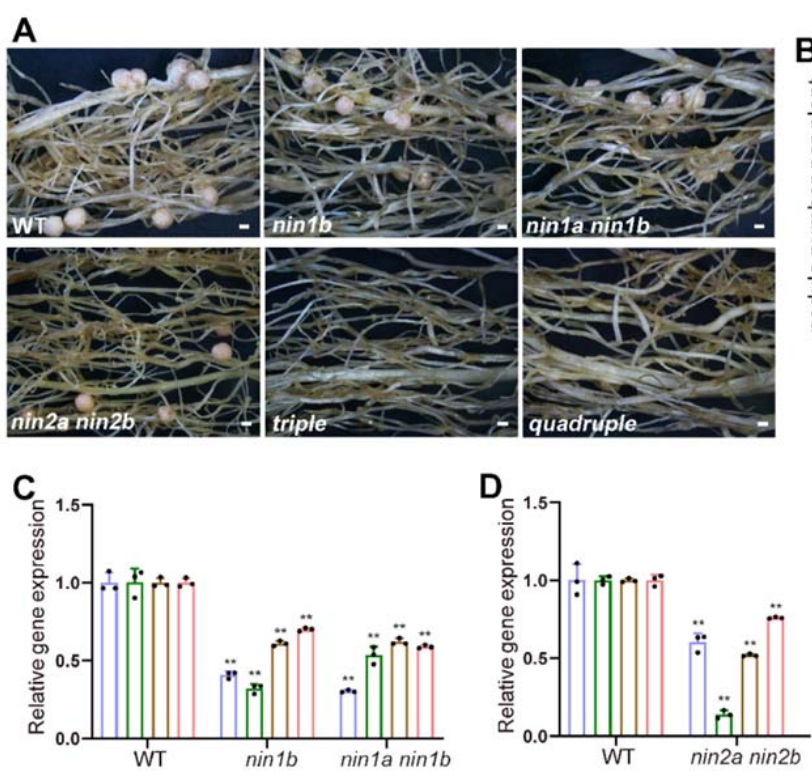
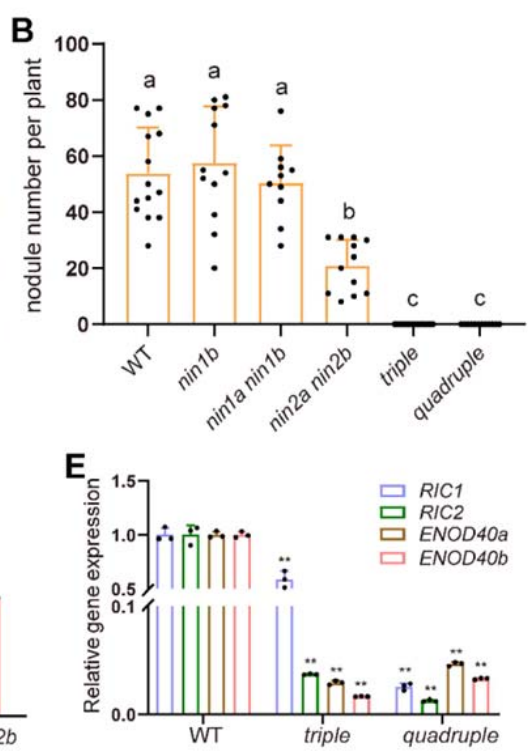

Figure 3. Nodulation phenotypes of Gmnin CRISPR-Cas9 mutants and gene expression levels in these mutants. A, Nodule phenotypes of G. max wild type (Huachun 6) and Gmnin CRISPR-Cas9 mutants 3 weeks after inoculation of B. japonicum USDA110. Sale bars $=1 \mathrm{~mm}$. B, Nodule numbers of G. max wild type and Gmnin CRISPR-Cas9 mutants at 3 wpi. Error bars show SE, dots represent individual nodule numbers. Data from one representative experiment of two or three independent experiments. The different letters indicate significant differences (one -way ANOVA-multiple comparisons). C to E, RT-qPCR analyze of the relative expression levels of symbiotic reporter genes in wild type and Gmnin mutants. Error bars indicate SE. Data from one representative experiment of three biological replicates. Asterisks indicate significant differences (Student's $t$-test, ${ }^{*} P<0.05,{ }^{*} P<0.01$, comparisons between WT control and experimental groups).

221 including infection foci and infection threads can be produced 7 days after inoculation

222 (Figure 4, E-H and M). Gmnin1a nin2a nin2b and Gmnin1a nin1b nin2a nin2b 223 showed excessive root hair curling in response to rhizobia (Figure 4, C and D), no 224 infection threads developed, and very rarely a few infection foci formed (Figure 4, K 225 and L). Surprisingly, Gmnin $2 a$ nin $2 b$ showed hype infection phenotype, produce more 226 infection foci and infection threads than wild type (Figure 4, I-J and N). Based on 

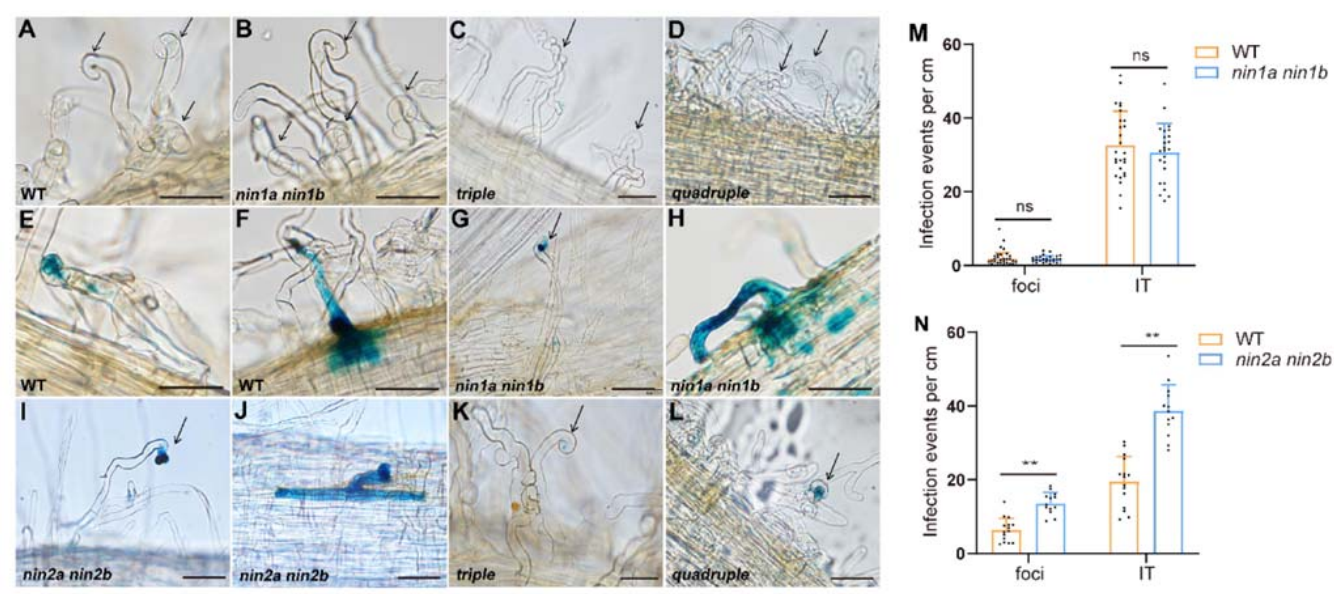

Figure 4. Root hair deformation and infection phenotypes of Gmnin mutants. A to D, Root hair deformation phenotypes of wild type (A), Gmninla nin1b (B), Gmninla nin2a nin2b (C) and Gmninla nin1b nin2a nin2b (D). E to L, Wild type (E, F), Gmninla nin1b (G, H) and Gmnin2a $\operatorname{nin} 2 b(\mathrm{I}, \mathrm{J})$ plants have normal infection foci $(\mathrm{E}, \mathrm{G}$ and $\mathrm{I})$ and infection threads $(\mathrm{F}, \mathrm{H}$ and $\mathrm{J})$, but Gmnin1a nin2a $\operatorname{nin} 2 b(\mathrm{~K})$ and Gmnin1a nin $1 b \operatorname{nin} 2 a \operatorname{nin} 2 b(\mathrm{~L})$ only occasionally developed a few infection foci after rhizobia inoculation. Arrowheads represent deformed root hairs. Scale bars $=$ $100 \mu \mathrm{m} . \mathrm{M}$ to $\mathrm{N}$, Infection events in wild type and Gmninla nin1b (M), Gmnin2a nin2b (N) 7 days after inoculation of B. japonicum USDA110 carrying a GUS reporter gene. Error bars show SE, dots represent individual numbers. Data from one representative experiment of two independent experiments. Foci: infection foci; IT: infection thread. ns: no significant difference (Student's $t$-test). these results we concluded that the Gmnin triple or quadruple mutants have similar phenotypes as reported for Ljnin and Mtnin mutants, which showed excessive root hair deformation, developed occasional infection foci, and were unable to form nodules. Nodule Primordium-Like Structures 

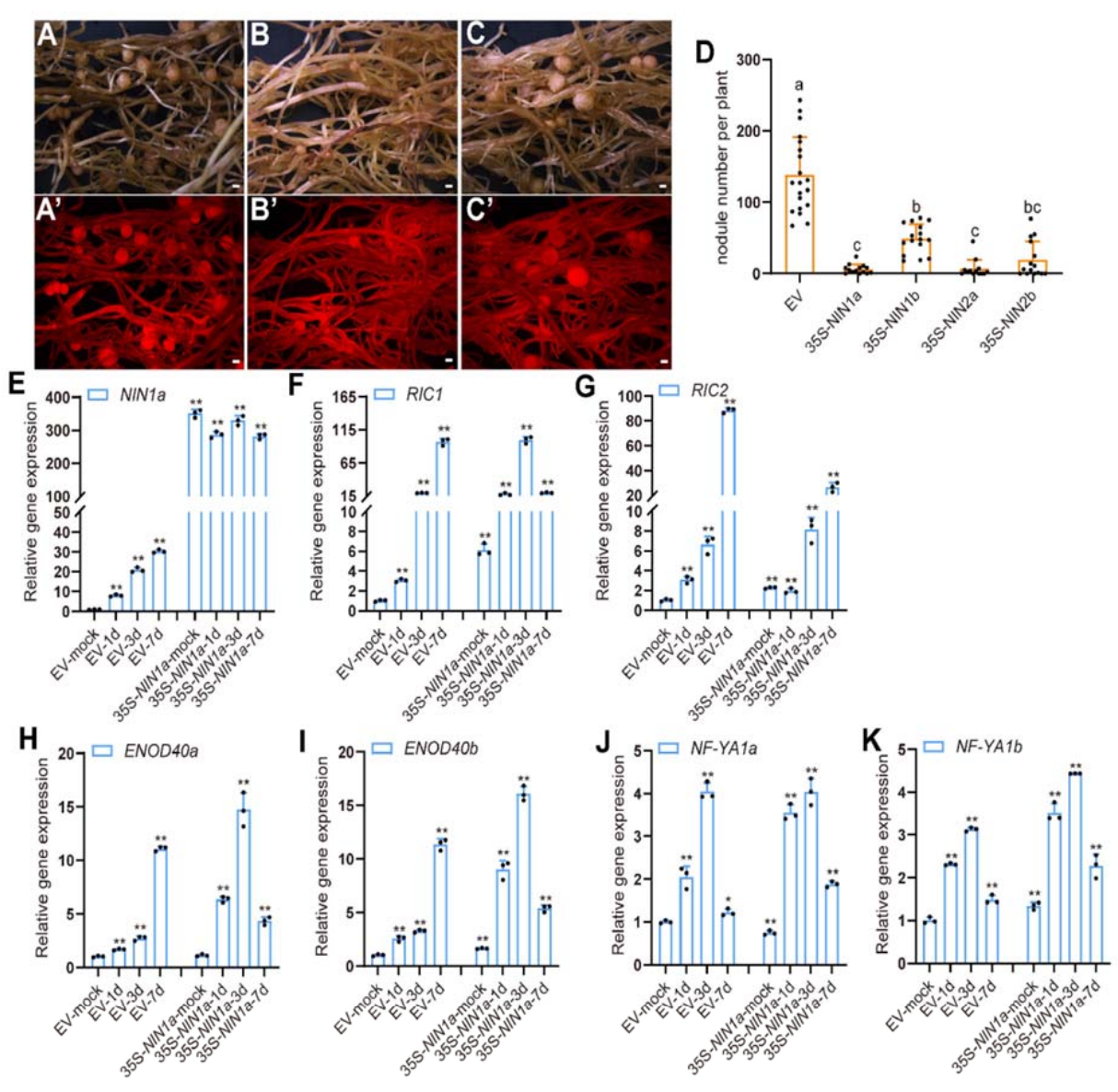

Figure 5. GmNIN overexpression lines suppress nodulation. A to C, Nodule phenotypes of EV (A), p35S: GmNINIa (B) and p35S: GmNINIb (C) lines at 3 wpi. Scale bars= $1 \mathrm{~mm}$. Upper panels (A-C) represent bright-field images, and the lower panels (A'-C') represent fluorescence images show mCherry expression in the same transgenic roots. D, Nodule numbers of GmNIN overexpression transgenic roots at 3 wpi with B. japonicum. Error bars show SE, dots represent individual numbers. Data from one representative experiment of three independent experiments. Different letters indicate significant differences (one-way ANOVA-multiple comparisons). E to K, RT-qPCR analyze transcription levels of GmNIN1a (E), RIC1 (F), RIC2 (G), ENOD40a (H), ENOD $40 b(\mathrm{I}), N F-Y A l a(\mathrm{~J})$ and $N F-Y A l b(\mathrm{~K})$ in EV and GmNINIa-OE hairy roots after rhizobia inoculation. Error bars indicate SE. Data from one representative experiment of three biological replicates. Asterisks indicate significant differences (Student's $t$-test, ${ }^{*} P<0.05,{ }^{* *} P<0.01$, comparisons between EV control and GmNINla-OE hairy roots). 
236 root nodule-like structures in the absence of rhizobia inoculation (Soyano et al., 2013;

237 Vernie et al., 2015). We then overexpressed GmNINs using 35S promoter driven

$238 G m N I N$ cDNA in G. $\max$ hairy roots. The transgenic roots were inoculated with

239 rhizobia and the nodulation phenotypes were scored at 21 dpi with B. japonicum. We

240 found that overexpression of GmNIN1 $a$, GmNIN2 $a$ or GmNIN2b dramatically reduced

241 nodule numbers; while with overexpression GmNIN1b, the nodule numbers were less

242 than EV control but more than GmNIN1a and GmNIN2a overexpression lines (Figure

2435 , A-D). The expression of symbiotic reporter genes were examined in the GmNIN1a

244 overexpression roots after rhizobia inoculation. The results showed that GmNIN1a

245 was highly induced after rhizobia inoculation and in GmNIN1a overexpression hairy

246 roots (Figure 5E). Symbiotic reporter genes such as RIC1, RIC2, ENOD40 and

$247 \quad N F-Y A 1$ were induced by rhizobia in the EV control and the GmNIN1a overexpression

248 transgenic roots (Figure 5, F-K).

249 We next examined whether overexpression of GmNINs can induce the formation 250 of nodule primordium-like structures without rhizobia inoculation. The composite 251 plants with transgenic hairy roots were transferred intro vermiculite: perlite mixture 252 (1:1) and the phenotype was scored 35 days after transfer. We found that 253 overexpression of GmNIN1a, GmNIN2a and GmNIN2b produced abnormal root 254 growths, including white nodule-like structures, small bumps and short but enlarged 255 lateral roots (Figure 6, A-D, I and Supplemental Figure S8, C-H). Nearly all the 256 GmNIN1 a overexpression roots, $60 \%$ of GmNIN2a overexpression roots, and $80 \%$ of 257 GmNIN2b overexpression roots showed these abnormal architectures, but none of EV 258 and GmNIN1b overexpression roots developed this kind of malformed structures, in 259 three independent experiments (Figure 6I and Supplemental Figure S8, A-H). 260 Sectioning of the GmNINs-induced abnormal structures revealed that the nodule-like 261 structures and white bumps were formed via cortical cell division and were 262 anatomically similar to root nodule primordia (Figure 6, E-F and Supplemental Figure 263 S8, I-J, M-N); the malformed nodule-like structures have central vascular systems 264 similar to lateral roots but also have undergone extensive cortical cell divisions and 

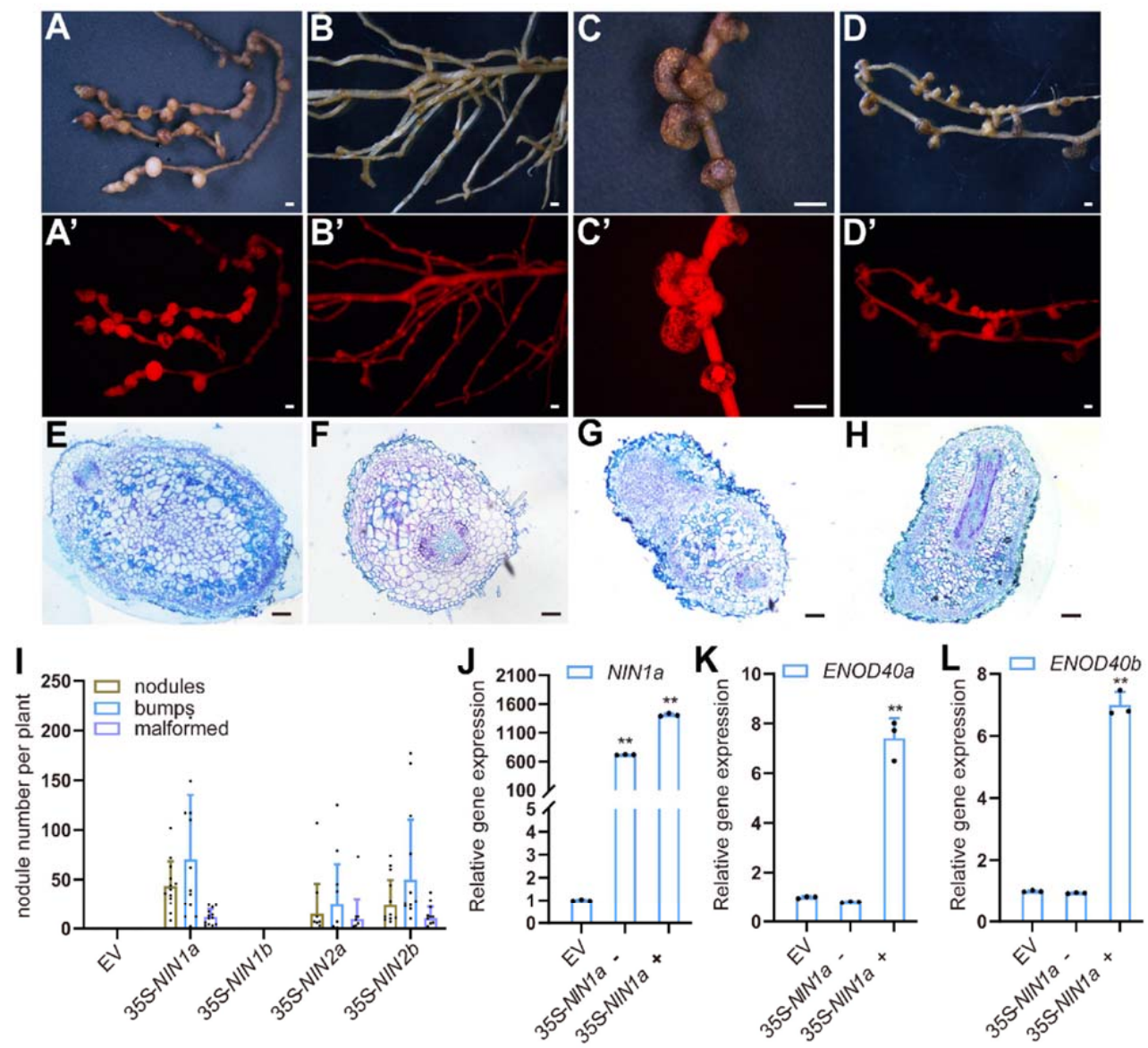

Figure 6. GmNINs overexpression triggers the formation of spontaneous nodule-like structures. A to D, p35S: GmNINla expressed in wild type hairy roots and cultured for 5 weeks without rhizobia inoculation, produced nodules (A), small white bumps (B) and malformed nodule-like structures (C and D). Upper panels (A-D) showed bright-field images, and lower panels (A'-D') are epifluorescence microscopy images show mCherry expression in the same transgenic roots. Scale bars $=1 \mathrm{~mm}$. E to $\mathrm{H}$, Transverse sections of spontaneous nodules $(\mathrm{E})$, small white bumps (F) and malformed nodule-like structures $(\mathrm{G}$ and $\mathrm{H})$ in GmNINla-OE hairy roots in the absence of rhizobia inoculation. Scale bars $=100 \mu \mathrm{m}$. I, Nodule numbers in the overexpressed GmNINs hairy roots 5 weeks after culture. Dots represent individual numbers. Data from one representative experiment of three independent experiments. J to L, RT-qPCR analyze transcription levels of GmNINla (J), ENOD40a (K) and ENOD40b (L) in EV and p35S:GmNINIa hairy roots. $p 35 S$ : NINI $a$ - represent no spontaneous nodule-like structures hairy roots, and p35S: NINla + represent have spontaneous nodule-like structures hairy roots. Error bars indicate SE. Data from one representative experiment of three biological replicates. Asterisks indicate significant differences (Student's $t$-test, $* P<0.05,{ }^{*} P<0.01$, comparisons between EV control and GmNINIa-OE hairy roots).

show over-proliferation of pericycle and cortical cells (Figure 6, G-H and

266 Supplemental Figure S8, K-L, O-P). RT-qPCR analysis showed that GmNIN1a was 
267 highly expressed in the transgenic hairy roots, but the early nodulin gene ENOD40 268 was highly expressed in the roots showing abnormal architecture, while was not 269 changed in normal roots (Figure 6, J-L).

270 LjNIN systemically suppresses nodulation through the AON pathway (Soyano et 271 al., 2014). We expressed 35S:GmNIN1a in wild type or nark mutant plants, and the 272 nodulation phenotype was analyzed in transformed or untransformed hairy roots at 21 273 dpi. The mean number of nodules was significantly suppressed in both 274 mCherry-positive transgenic and mCherry-negative untransformed roots in wild type 275 background, but the repressive level was stronger in mCherry-positive transgenic 276 roots (Figure 7, A-B and E). However, the suppression effect was not present in nark 277 mutant background either in transgenic or non-transgenic hairy roots (Figure 7, C-D 278 and E). This result indicates that ectopic expression of GmNIN1a systemically inhibits 279 nodulation via the AON pathway. 

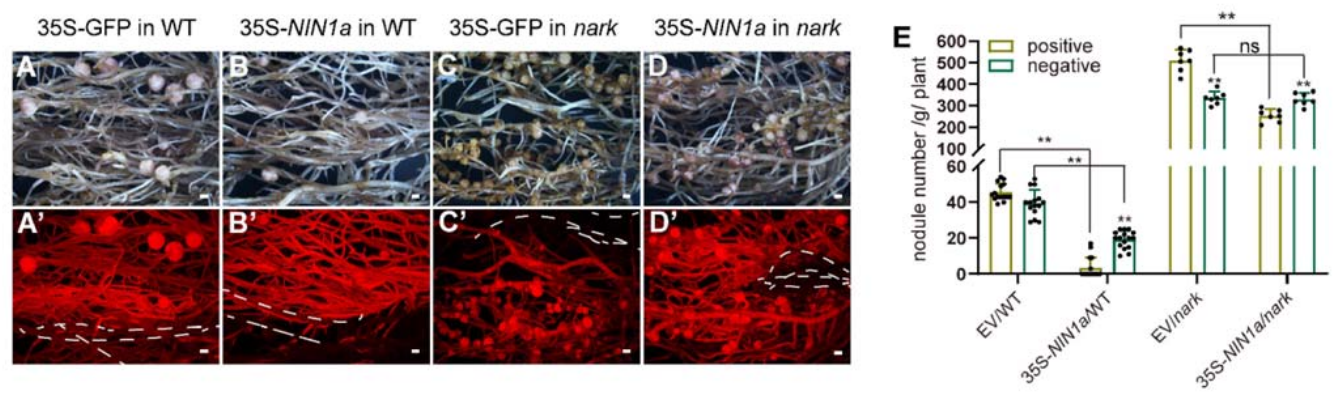

Figure 7. Systemic inhibition of nodulation by overexpression of GmNIN1a. Ato D, Nodulation on mCherry-negative untransformed roots and mCherry-positive transformed roots on wild type carrying EV (A) or p35S-GmNINla (B), and on nark carrying EV (C) or p35S-GmNINla (D) at 3 wpi. Upper panels (A-D) show composite bright-filed images. Lower panels (A'-D') show corresponding fluorescent images with mCherry as a transformation marker for transgenic hairy roots. White dash in lower panels represent mCherry-negative roots. Scale bars $=1 \mathrm{~mm}$. E, Nodule numbers on mCherry-positive and -negative roots that were transformed with the EV or p35S-GmNIN1a in wild type (WT) or nark mutants. Dots represent individual numbers. Asterisks indicate significant differences between mCherry-negative untransformed roots and mCherry-positive transformed roots on wild type carrying EV or $p 35 S$-GmNIN1a (Student's $t$-test, $* P<0.05, * * P<0.01$, ns: no significant difference).

282

283

284

285

286

287

288

289

290

291

292

293

294

295

\section{DISCUSSION}

Soybean is an ancient polyploid due to genome duplications, resulting in a nearly $75 \%$ of genes being present in multiple copies (Schmutz et al., 2010). This makes it more difficult to identify recessive inheritance in this duplicated genome. Using a CRISPR-Cas9 approach, we were able to make higher order mutants, and then analyzed their biological function. NIN is essential for all processes in root nodule formation, and G. max contains four NIN genes due to genome duplications, but which genes are required for nodulation was not studied. In this work, we carefully examined GmNIN expression patterns and nodulation phenotypes in CRISPR-Cas9 mutants or GmNIN overexpression lines. The results suggested that GmNIN1a, GmNIN2a and GmNIN2b are essential for nodulation in a functionally redundant manner, but that GmNIN1b likely has only a minor role in root nodule symbiosis.

NIN is specifically induced and expressed in nodules (Marsh et al., 2007; Schauser et al., 1999). In G. max, all these four GmNINs were induced by rhizobia 
and were highly expressed in nodules. In all these GmNIN promoters, contains the CYC motif, but the most important $2^{\text {nd }}$ CE motif was not conserved in GmNIN1b promoter. The CYC motif is required for NIN's expression in epidermal cells where infection threads are initiated. Compared with $\mathrm{p} M t N I N_{C Y C}$ GUS, which is expressed in epidermal and pericycle cells (Liu et al., 2019), pGmNIN2aCYC:GUS was only detected in epidermal cells and nodules. This difference might result from shorter promoter we used, and the GUS expression was weaker than detective. Or this might also suggests that another motif in NIN promoter besides the CYC motif may be required for NIN expression in pericycle cells. However, pGmNIN2 $a_{C E-C Y C}$ GUS was found to express in epidermal cells, pericycle cells, divided cortical cells, and in nodules, suggesting that this conserved $2^{\text {nd }} \mathrm{CE}$ motif is essential and sufficient for GmNIN2 $a$ expressed in pericycle cells.

Compared with other species, G. max contains four NIN genes and all of them were induced and expressed in nodules. Several studies including ours, showed that RNAi knock down GmNIN1a showed a nodule-minus phenotype (He et al., 2020 and this study), suggesting that GmNINla is sufficient for NIN's function in root nodule symbiosis. However, after careful analysis of GmNIN expression levels in GmNIN1a RNAi plants, we found that not only the expression of GmNINla, but also the expression of other three GmNINs, was reduced, suggesting that our GmNIN1a RNAi plants phenocopy a GmNIN quadruple mutant. Moreover, knock down other GmNINs reduced nodule numbers, and gene expression showed these RNAi lines, also knock down other GmNINs. Our RNAi analysis of GmNINs suggest that they act in a functionally redundant manner to regulate the root nodule symbiosis. To further validate this hypothesis, we made a series of GmNINs mutants using a CRISPR-Cas9 approach. Unexpectedly, we found that Gmnin1b and Gmnin1a nin1b showed a wild type nodulation phenotype, including root hair deformation, rhizobial infection and nodule numbers. Gmnin2a nin $2 b$ mutant exhibited an intermediate phenotype with reduced nodule number. Gmnin $1 a$ nin $2 a$ nin $2 b$ triple and Gmnin1a nin1b nin2a nin $2 b$ quadruple mutants showed a typical Ljnin and Mtnin nodulation phenotype, including 
325

326

327

328

329

330

331

332

333

334

335

336

337

338

339

340

341

342

343

344

345

346

347

348

349

350

351

352

excessive root hair deformation, nearly no rhizobial infection events and no nodules.

These results suggested that GmNIN1a, GmNIN2a, GmNIN2b are required for nodule organogenesis in a dosage dependent manner, while GmNIN1b is not able to compensate their function in Gmnin 1 a nin $2 a \operatorname{nin} 2 b$ triple mutant.

Unexpectedly, Gmnin $2 a \operatorname{nin} 2 b$ mutant showed a hyper infection phenotype that was similar to Ljdephne or Mtdephne-like mutant (Yono et al., 2014; Liu et al., 2019). Considering the non-infection phenotype in triple and quadruple mutants and normal infection in Gmnin1a nin $1 b$ double mutant, it is less likely that GmNIN2a and GmNIN2b simply play a negative role in rhizobial infection. One possibility is that the GmNINs expressions at cellular level might be differentiated with diversified biological implications. For instance, we speculate that GmNIN2a and GmNIN2b in nodule cortex cells might non-cell-autonomously modulate GmNIN1a expression in epidermal cells. In Gmnin2a nin2b mutant, GmNIN1a in epidermal cells might be induced and lead to more infection events. So far, we failed to clone GmNIN1a promoter for detailed expression analysis, and further studies are required to validate these hypotheses. Taken together, our results show that GmNINs play functionally redundant yet complicated roles in the root nodule symbiosis.

Due to multiple genome duplications, approximately $75 \%$ of soybean genes are duplicated leading to the perspective of high genetic redundancy (Schmutz et al., 2010). In some cases, duplicated genes are asymmetrically redundant, as mutation in one gene may cause a phenotype while in the other gene showed no or weak phenotype (Vaddepalli et al., 2019). Such asymmetric genetic redundancy is usually explained by diverged or unequal gene expression (Vaddepalli et al., 2019). Here we show that GmNIN1b plays a minor role in root symbiosis, even though its expression is comparable to the other three GmNIN homologs, but the most important $2^{\text {nd }} \mathrm{CE}$ region was not conserved in GmNIN1b, implying its expression pattern may different with other NINs. Such divergence may also be attributed to variations in protein sequences, GmNIN1b was shorter than other GmNINs, and overexpression of 
$353 G m N I N 1 b$ lead to marginal phenotypes. Elucidation of the protein sequences among 354 GmNIN homologs may provide further sights into the evolution of NIN genes in 355 legumes.

356 Consistent with LjNIN, overexpression of GmNINs reduced nodule numbers 357 after rhizobia inoculation, and this suppression nodulation was systemic and 358 dependent on AON pathway (Soyano et al., 2013; Soyano et al., 2014). However, $359 G m N I N 1 a$ and its putative target genes (e.g. GmRIC1, GmRIC2 and GmNF-YA1) were 360 also induced in GmNIN1a overexpression plants. This result was different with LjNIN 361 ectopic expression which down-regulated NIN expression and NIN activity (Soyano 362 et al., 2014). This difference might have resulted from our use of the constitutive $35 \mathrm{~S}$ 363 promoter to drive GmNIN1a expression, rather than the DEX-inducible expression 364 system used for the study of LjNIN. Moreover, we found that the overexpression of $365 G m N I N 1 a$, GmNIN2a or GmNIN2b, but not GmNIN1b, can form spontaneous 366 malformed root nodule-like structures in the absence of rhizobia inoculation. We 367 noted some cases where the structures that formed appeared to resemble hybrids 368 between lateral roots and nodules, with proliferation of cortical cells around a central 369 vascular bundle. The formation of nodules with central vascular bundles has been 370 reported in several plant and rhizobial nodulation mutants (Imaizumi-Anraku et al., 371 2000; Guan et al., 2013), and better understanding of this phenomenon might give 372 insights into how the peripheral vasculature particular to nodules forms. All these 373 results support that GmNINs have similar important roles in root nodule symbiosis, 374 and GmNIN1a, GmNIN2a and GmNIN2b function redundantly to regulate 375 nodulation.

\section{MATERIAL AND METHODS} was used in this study. nark mutant in Huachun 6 genetic background was previously 
381 generated by CRSIPR-Cas9 (Bai et al., 2020). For hairy root transformation,

382 Agrobacterium rhizogenes strain K599 was used. A. tumefaciens strain EHA105 was

383 used for expressing in N. benthamiana, and A. tumefaciens strain GV3101 was used

384 for generating CRISPR-Cas9 stable mutants. Plasmids were transformed into

385 Escherichia coli DH10B or DH5a for cloning.

387 Analysis of Promoter of GmNINs

388 The sequence of GmNINs' promoters were extracted from Phytozome 12.1 (http:// 389 phytozome.jgi.doe.gov/pz/portal.html) and multiple alignment using website (http:// 390 www.ebi.ac.uk/Tools/msa/clustalo/) and the conserved regions and putative 391 CYCLOPS binding site in GmNINs' promoters were searched in their promoter 392 regions.

393 The GmNIN2a CE and CYC were amplified from Williams 82 leaf genomic 394 DNA as template, the primer sequences are shown in Table S3. The PCR products and 395 binary vector pK7FWG2-R which was modified by adding GUS gene before GFP, 396 then using ClonExpress ${ }^{\circledR}$ multis One Step Cloning Kit (Vazyme), to generate

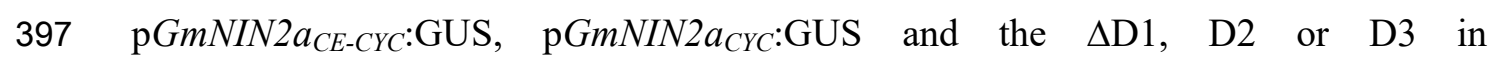
398 pGmNIN $2 a_{C E-C Y C}$ :GUS. The constructs were confirmed by DNA sequencing and 399 expressed in A. rhizogenes strain K599 for hairy root transformation. The transgenic 400 roots were transferred into a vermiculite:perlite mixure $(1: 1)$ and inoculated with $B$. 401 japonicum USDA110 5-7 days after transfer. The roots were harvested for GUS 402 staining at indicated timepoints after inoculation. The stained nodules were sectioned 403 and images were taken by light microscope (Nikon Eclipse Ni).

406 The GmNINs cDNA were amplified from G. max Williams 82 root cDNA using the 
407 primers shown in Table S2. The PCR products were cloned into pDONR207 and then 408 recombined into the vector pK7WGF2 to generate $35 S-G m N I N s$, and then recombined 409 this product into pUb-3xflag and p35S:mCherry (in order to replace its pUb promoter).

410 The constructs were confirmed by DNA sequencing and transformed into $A$. 411 tumefaciens strain EHA105, then co-infiltrated into $N$. benthamiana leaves plus p19.

412 The images were taken by confocal microscopy with DAPI staining and protein 413 expression levels were analyzed by western blot $60 \mathrm{hrs}$ after infiltration.

\section{RNAi and Overexpression of GmNINs}

416 For knock down GmNINs by RNAi strategy, specific fragments of GmNINs (about 417 150-200 bp) were amplified by PCR using G. max cDNA as template. The PCR 418 products were cloned into pDONR207 and then recombined into pUb-GWS-GFP. For 419 overexpression GmNINs, full-lengths GmNINs were amplified by PCR using G. max 420 cDNA as template and cloned into pDONR207, then recombined into pK7WGF2. The 421 primers used are showed in Table S3 and all the constructs in pDONR207 were 422 sequenced. These constructs were introduced into A. rhizogenes K599 by 423 electroporation and then expressed in G. $\max$ Williams 82 by hairy root 424 transformation. The transgenic hairy roots were planted into a vermiculite:perlite $(1: 1)$ 425 mixture and inoculated by B. japonicum USDA110 5-7 days after transfer. GmNINs 426 overexpression hairy roots also were planted in a vermiculite:perlite (1:1) mixture 427 without rhizobial inoculation. And the phenotypes were analyzed at the indicated time 428 points.

\section{Generation of Gmnin CRISPR-Cas9 Mutants}

431 To generate Gmnin mutants by CRISPR-Cas9, two small-guide RNAs targeting 432 different regions of GmNINs were designed and cloned into the pGES201 vector. The 433 pooled constructs were introduced into A. tumefaciens strain GV3101, which was then 
434 transformed into soybean cultivar Huachun 6 by Agrobacterium-mediated 435 transformation as described (Bai et al., 2020). The mutant genotypes were identified 436 using the Hi-TOM platform (Liu et al., 2019).

437 To observe Gmnin CRISPR-Cas9 infection phenotype, the mutants seedling were 438 grown in a vermiculite:perlite mixture (1:1) and inoculated with B. japonicum 439 USDA110 contains GUS reporter. The inoculated roots were harvested 7 days or 21 440 days after inoculation and the roots were stained by GUS (7 dpi), and phenotypes 441 were observed under a light microscope (Nikon Eclipse Ni).

\section{Gene Expression Analysis}

444 The G. max roots were harvested and total RNA was extracted using an RNAprep

445 Plant plus Kit (Tiangen), and $1^{\text {st }}$ strand cDNA was synthesized using Transcript

446 One-Step gDNA Removal and cDNA Synthesis super Mix Kit (TransGen Biotech.).

447 RT-qPCR was performed on three biological replicates using an ABI StepOne PCR

448 detection system with SYBR Green (Takara). GmUbiquitin (SUBI-2.2) expression

449 was used as an internal control.

\section{$451 \quad$ Statistical Analysis}

452 Statistical significance was analyzed by Student's $t$-test $\left(* P<0.05,{ }^{*} P<0.01\right)$ or

453 one way ANOVA (non-parametric or mixed) and error bars indicate SE.

454 Histograms were generated using GraphPad Prism 8.0 software.

455

\section{ACKNOWLEDGMENTS}

457 We thank Prof. Min Wei (Lanzhou U. China) for providing USDA110 carrying GUS 458 strain, and Prof. Jeremy Murray (CEMPS, China) for helpful editing on the 
459

460

461

462

463

464

465

466

467

468

469

470

471

472

473

474

475

476

477

478

479

480

481

482

483

484

manuscript. This work was supported by CAS grant (ZDRW-ZS-2019-2) and the

National Key Research and Development Program of China (2016YFD0100702).

\section{CONFLICTS OF INTEREST}

The authors declare that they have no conflicts of interest.

\section{Figure Legends}

Figure 1. GmNINs were induced in soybean roots and nodules by rhizobia inoculation and have nodulation-specific expression patterns. Ato $\mathrm{H}$, Expression levels of GmNIN1a (A, E), GmNIN1b (B, F), GmNIN2a (C, G) and GmNIN2b (D, H) in $G$. $\max$ roots (A-D) and nodules (E-H) after rhizobia inoculation. RT-qPCR were used to measure gene expression levels, normalized relative to uninoculated roots $(0 \mathrm{~d})$. Values are means $\pm \mathrm{SE}$. Data shown are from one representative experiment of three biological replicates. Asterisks indicate significant differences between treated and control conditions ( $0 \mathrm{~d}$ ) (Student's $t$-test, * $P<0.05$, ** $P<0.01$ ). I to $\mathrm{R}$, The $p G m N I N 2 a_{C Y C}$ GUS (I-K), $p G m N I N 2 a_{C E-C Y C}$ :GUS (L-R) were expressed in G. max hairy roots, and the images were taken at 3 days (I, L), 5 days (M) or 14 days (J, N) after rhizobia inoculation. (K, O-R) Section of these nodules stained with ruthenium red. Arrows $(\mathrm{O})$ indicate GUS expression in pericycle cells. Scale bars: $1 \mathrm{~mm}(\mathrm{~J}, \mathrm{M}$ and $\mathrm{N}) ; 100 \mu \mathrm{m}(\mathrm{K}, \mathrm{Q}$ and $\mathrm{R}) ; 50 \mu \mathrm{m}(\mathrm{I}, \mathrm{L}, \mathrm{O}$ and $\mathrm{P})$.

Figure 2. Nodulation phenotypes in GmNIN RNAi hairy roots. A to E, Nodule phenotypes of EV control (A), GmNIN1a (B), GmNIN1b (C), GmNIN2a (D) and GmNIN2b (E) RNAi transgenic roots 3 weeks after rhizobia inoculation. Upper panels (A-E) show bright-field images and the lower panels (A'-E') are epifluorescence 
485 microscopy images showing GFP expression in the same transgenic roots. Scale bars $486=1 \mathrm{~mm}$. F, Nodule numbers of the EV and RNAi transgenic lines. The nodule 487 numbers were scored at 3 weeks post inoculation with B. japonicum USDA110. Data 488 from one representative experiment of three biological replicates. Error bars indicate 489 SE, dots represent individual nodule numbers. The different letters indicate significant 490 differences (one-way ANOVA-multiple comparisons). G to J, RT-qPCR analyze of 491 the relative expression levels of $\operatorname{GmNINs}(\mathrm{G}$ and $\mathrm{H})$ and symbiotic reporter genes (I 492 and J) in EV and GmNINs RNAi transgenic roots at 7 days post inoculation with $B$. 493 japonicum USDA110. Error bars indicate SE. Data shown are from one representative 494 experiment using three biological replicates. Asterisks indicate significant differences $495\left({ }^{*} P<0.05,{ }^{* *} P<0.01\right.$, Student's $t$-test, comparisons between EV control and 496 experimental groups).

497 Figure 3. Nodulation phenotypes of Gmnin CRISPR-Cas9 mutants and gene 498 expression levels in these mutants. A, Nodule phenotypes of $G$. max wild type 499 (Huachun 6) and Gmnin CRISPR-Cas9 mutants 3 weeks after inoculation of $B$. 500 japonicum USDA110. Sale bars= $1 \mathrm{~mm}$. B, Nodule numbers of $G$. max wild type and 501 Gmnin CRISPR-Cas9 mutants at 3 wpi. Error bars show SE, dots represent individual 502 nodule numbers. Data from one representative experiment of two or three 503 independent experiments. The different letters indicate significant differences (one 504 -way ANOVA-multiple comparisons). C to E, RT-qPCR analyze of the relative 505 expression levels of symbiotic reporter genes in wild type and Gmnin mutants. Error 506 bars indicate SE. Data from one representative experiment of three biological 507 replicates. Asterisks indicate significant differences (Student's $t$-test, $* P<0.05, * * P$ $508<0.01$, comparisons between WT control and experimental groups).

509 Figure 4. Root hair deformation and infection phenotypes of Gmnin mutants. A to D, 510 Root hair deformation phenotypes of wild type (A), Gmninla nin1b (B), Gmnin1a $511 \operatorname{nin} 2 a \operatorname{nin} 2 b(\mathrm{C})$ and Gmnin1a nin1b $\operatorname{nin} 2 a \operatorname{nin} 2 b$ (D). E to L, Wild type (E, F), $512 \operatorname{Gmnin} 1 a \operatorname{nin} 1 b(\mathrm{G}, \mathrm{H})$ and Gmnin2a $\operatorname{nin} 2 b(\mathrm{I}, \mathrm{J})$ plants have normal infection foci (E, 
$513 \mathrm{G}$ and $\mathrm{I}$ ) and infection threads (F, H and $\mathrm{J}$ ), but Gmin1a $\operatorname{nin} 2 a \operatorname{nin} 2 b(\mathrm{~K})$ and 514 Gmnin1a nin1b $\operatorname{nin} 2 a \operatorname{nin} 2 b$ (L) only occasionally developed a few infection foci 515 after rhizobia inoculation. Arrowheads represent deformed root hairs. Scale bars = $516100 \mu \mathrm{m}$. M to N, Infection events in wild type and Gmninla nin1b (M), Gmnin2a $517 \operatorname{nin} 2 b(\mathrm{~N}) 7$ days after inoculation of $B$. japonicum USDA110 carrying a GUS 518 reporter gene. Error bars show SE, dots represent individual numbers. Data from one 519 representative experiment of two independent experiments. Foci: infection foci; IT: 520 infection thread. ns: no significant difference (Student's $t$-test).

521 Figure 5. GmNIN overexpression lines suppress nodulation. A to C, Nodule 522 phenotypes of EV (A), p35S: GmNIN1a (B) and p35S: GmNIN1b (C) lines at 3 wpi. 523 Scale bars $=1 \mathrm{~mm}$. Upper panels (A-C) represent bright-field images, and the lower 524 panels (A'-C') represent fluorescence images show mCherry expression in the same 525 transgenic roots. D, Nodule numbers of GmNIN overexpression transgenic roots at 3 526 wpi with B. japonicum. Error bars show SE, dots represent individual numbers. Data 527 from one representative experiment of three independent experiments. Different 528 letters indicate significant differences (one-way ANOVA-multiple comparisons). E to $529 \mathrm{~K}, \mathrm{RT}-\mathrm{qPCR}$ analyze transcription levels of $\operatorname{GmNIN1a}(\mathrm{E}), \operatorname{RIC1}(\mathrm{F}), \operatorname{RIC} 2(\mathrm{G})$, 530 ENOD40a (H), ENOD40b (I), NF-YAla (J) and NF-YAIb (K) in EV and 531 GmNIN1a-OE hairy roots after rhizobia inoculation. Error bars indicate SE. Data 532 from one representative experiment of three biological replicates. Asterisks indicate 533 significant differences (Student's $t$-test, $* P<0.05, * * P<0.01$, comparisons between 534 EV control and GmNINIa-OE hairy roots).

535 Figure 6. GmNINs overexpression triggers the formation of spontaneous nodule-like 536 structures. A to D, p35S: GmNIN1a expressed in wild type hairy roots and cultured 537 for 5 weeks without rhizobia inoculation, produced nodules (A), small white bumps 538 (B) and malformed nodule-like structures (C and D). Upper panels (A-D) showed 539 bright-field images, and lower panels (A'-D') are epifluorescence microscopy images 540 show mCherry expression in the same transgenic roots. Scale bars $=1 \mathrm{~mm}$. E to $\mathrm{H}$, 
541 Transverse sections of spontaneous nodules (E), small white bumps (F) and

542 malformed nodule-like structures $(\mathrm{G}$ and $\mathrm{H})$ in GmNINIa-OE hairy roots in the

543 absence of rhizobia inoculation. Scale bars $=100 \mu \mathrm{m}$. I, Nodule numbers in the

544 overexpressed GmNINs hairy roots 5 weeks after culture. Dots represent individual

545 numbers. Data from one representative experiment of three independent experiments.

$546 \mathrm{~J}$ to L, RT-qPCR analyze transcription levels of GmNIN1a (J), ENOD40a (K) and

$547 E N O D 40 b(\mathrm{~L})$ in EV and p35S:GmNIN1a hairy roots. p35S: NIN1a - represent no

548 spontaneous nodule-like structures hairy roots, and p35S: NIN1a + represent have

549 spontaneous nodule-like structures hairy roots. Error bars indicate SE. Data from one

550 representative experiment of three biological replicates. Asterisks indicate significant

551 differences (Student's $t$-test, $* P<0.05$, ${ }^{* *} P<0.01$, comparisons between EV control

552 and GmNIN1a-OE hairy roots).

553 Figure 7. Systemic inhibition of nodulation by overexpression of GmNIN1a. Ato D,

554 Nodulation on mCherry-negative untransformed roots and mCherry-positive

555 transformed roots on wild type carrying EV (A) or p35S-GmNIN1a (B), and on nark

556 carrying EV (C) or p35S-GmNIN1a (D) at 3 wpi. Upper panels (A-D) show

557 composite bright-filed images. Lower panels (A'-D') show corresponding fluorescent

558 images with mCherry as a transformation marker for transgenic hairy roots. White

559 dash in lower panels represent mCherry-negative roots. Scale bars $=1 \mathrm{~mm}$. E, Nodule

560 numbers on mCherry-positive and -negative roots that were transformed with the EV

561 or p35S-GmNIN1a in wild type (WT) or nark mutants. Dots represent individual

562 numbers. Asterisks indicate significant differences between mCherry-negative

563 untransformed roots and mCherry-positive transformed roots on wild type carrying

$564 \mathrm{EV}$ or $p 35 S$-GmNIN1a (Student's $t$-test, ${ }^{*} P<0.05$, $* * P<0.01$, ns: no significant 565 difference). 


\section{Parsed Citations}

Bai M, Yuan J, Kuang H, Gong P, Li S, Zhan Z, Liu B, Sun J, Yang M, Yang L, Wang D, Song S, Guan Y (2020) Generation of a multiplex mutagenesis population via pooled CRISPR-Cas9 in soyabean. Plant Biotechnol J 18: 721-731

Google Scholar: Author Only Title Only Author and Title

Borisov AY, Madsen LH, Tsyganov VE, Umehara Y, Voroshilova VA, Batagov AO, Sandal N, Mortensen A, Schauser L, Ellis N, Tikhonovich IA Stougaard J (2003) The Sym35 gene required for root nodule development in pea is an ortholog of NIN from Lotus japonicus. Plant Physiol 131:1009-17

Google Scholar: Author Only Title Only Author and Title

Bu F, Rutten L, Roswanjaya YP, Kulikova O, Rodriguez-Franco M, Ott T, Bisseling T, van Zeijl A, Geurts R (2020) Mutant analysis in the nonlegume Parasponia andersonii identifies NIN and NF-YA1 transcription factors as a core genetic network in nitrogen-fixing nodule symbioses. New Phytol 226: 541-554

Google Scholar: Author Only Title Only Author and Title

Clavijo F, Diedhiou I, Vaissayre V, Brottier L, Acolatse J,Moukouanga D, Crabos A, Auguy F, Franche C, Gherbi H, Champion A, Hocher V, Barker D, Bogusz D, Tisa LS, Svistoonoff S (2015) The Casuarina NIN gene is transcriptionally activated throughout Frankia root infection as well as in response to bacterial diffusible signals. New Phytol 208: 887-903

Google Scholar: Author Only Title Only Author and Title

Griesmann M, Chang Y, Liu X, Song Y, Haberer G, Crook MB, Billault-Penneteau B, Lauressergues D, Keller J, Imanishi L, Roswanjaya YP, Kohlen W (2018) Phylogenomics reveals multiple losses of nitrogen-fixing root nodule symbiosis. Science 361

Google Scholar: Author Only Title Only Author and Title

Guan D, Stacey N, Liu C, Wen J, Mysore KS, Torres-Jerez I, Vernie T, Tadege M, Zhou C, Wang ZY, Udvardi MK, Oldroyd GE, Murray JD (2013) Rhizobial infection is associated with the development of peripheral vasculature in nodules of Medicago truncatula. Plant

Physiol 162: 107-115

Google Scholar: Author Only Title Only Author and Title

Hayashi S, Reid DE, Lorenc MT, Stiller J, Edwards D, Gresshoff PM, Ferguson BJ (2012) Transient Nod factor-dependent gene expression in the nodulation-competent zone of soybean (Glycine max [L.] Merr.) roots. Plant Biotechnol J 10: 995-1010

Google Scholar: Author Only Title Only Author and Title

He C, Gao H, Wang H, Guo Y, He M, Peng Y, Wang X (2020) GSK3-mediated stress signaling inhibits legume-rhizobium symbiosis by phosphorylating GmNSP1 in soybean. Mol Plant. https://doi.org/10.1016/j.molp.2020.12.015

Google Scholar: Author Only Title Only Author and Title

Imaizumi-Anraku H, Kouchi H, Syono K, Akao S, Kawaguchi M (2000) Analysis of ENOD40 expression in alb1, a symbiotic mutant of Lotus japonicus that forms empty nodules with incompletely developed nodule vascular bundles. Mol Gen Genet 264: 402-410

Google Scholar: Author Only Title Only Author and Title

Indrasumunar A, Gresshoff PM (2010) Duplicated Nod-Factor receptor 5 (NFR5) genes are mutated in soybean. Plant Signal Behav 5 : 535-6

Google Scholar: Author Only Title Only Author and Title

Indrasumunar A, Kereszt A, Searle I, Miyagi M, Li D, Nguyen CD, Men A, Carroll BJ, Gresshoff PM (2010) Inactivation of duplicated nod factor receptor 5 (NFR5) genes in recessive loss-of-function non-nodulation mutants of allotetraploid soybean (Glycine max L. Merr.). Plant Cell Physiol 51: 201-14

Google Scholar: Author Only Title Only Author and Title

Indrasumunar A Searle I, Lin MH, Kereszt A Men A Carroll BJ, Gresshoff PM (2011) Nodulation factor receptor kinase 1alpha controls nodule organ number in soybean (Glycine max L. Merr). Plant J 65: 39-50

Google Scholar: Author Only Title Only Author and Title

Indrasumunar A, Wilde J, Hayashi S, Li D, Gresshoff PM (2015) Functional analysis of duplicated Symbiosis Receptor Kinase (SymRK) genes during nodulation and mycorrhizal infection in soybean (Glycine max). J Plant Physiol 176: 157-68

Google Scholar: Author Only Title Only Author and Title

Kawaharada Y, Nielsen MW, Kelly S, James EK, Andersen KR, Rasmussen SR, Füchtbauer W, Madsen LH, Heckmann AB, Radutoiu S, Stougaard J (2017) Differential regulation of the Epr3 receptor coordinates membrane-restricted rhizobial colonization of root nodule primordia. Nat Commun 8: 14534

Google Scholar: Author Only Title Only Author and Title

Laffont C, Ivanovici A, Gautrat P, Brault M, Djordjevic MA, Frugier F (2020) The NIN transcription factor coordinates CEP and CLE signaling peptides that regulate nodulation antagonistically. Nat Commun 11: 3167

Google Scholar: Author Only Title Only Author and Title

Libault M, Farmer A, Brechenmacher L, Drnevich J, Langley RJ, Bilgin DD, Radwan O, Neece DJ, Clough SJ, May GD, Stacey G (2010) Complete transcriptome of the soybean root hair cell, a single-cell model, and its alteration in response to Bradyrhizobium japonicum infection. Plant Physiol 152: 541-52

Google Scholar: Author Only Title Only Author and Title 
Libault M, Joshi T, Takahashi K, Hurley-Sommer A, Puricelli K, Blake S, Finger, RE, Taylor CG, Xu D, Nguyen HT, Stacey G (2009) Large-scale analysis of putative soybean regulatory gene expression identifies a Myb gene involved in soybean nodule development. Plant Physiol 151: 1207-20

Google Scholar: Author Only Title Only Author and Title

Lin JS, Li X, Luo Z, Mysore KS, Wen J, Xie F (2018) NIN interacts with NLPs to mediate nitrate inhibition of nodulation in Medicago truncatula. Nat Plants 4: 1125

Google Scholar: Author Only Title Only Author and Title

Liu CW, Breakspear A, Guan D, Cerri MR, Jackson K, Jiang S, Robson F, Radhakrishnan GV, Roy S, Bone C, Stacey N, Rogers C, Trick M, Niebel A, Oldroyd GED, de Carvalho-Niebel F, Murray JD (2019) NIN acts as a network hub controlling a growth module required for rhizobial infection. Plant Physiol 179:1704-1722

Google Scholar: Author Only Title Only Author and Title

Liu CW, Murray JD (2016) The role of flavonoids in nodulation host-range specificity: an update. Plants (Basel) 5.

Google Scholar: Author Only Title Only Author and Title

Liu J, Rutten L, Limpens E, van der Molen T, van Velzen R, Chen R, Chen Y, Geurts R, Kohlen W, Kulikova O, Bisseling T (2019) A remote cis-regulatory region is required for NIN expression in the pericycle to initiate nodule primordium formation in Medicago truncatula. Plant Cell 31: 68-83

Google Scholar: Author Only Title Only Author and Title

Liu J, Rasing M, Zeng T, Klein J, Kulikova O, Bisseling T (2021) NIN is essential for development of symbiosomes, suppression of defense and premature senescence in Medicago truncatula nodules. New Phytol. doi: 10.1111/NPH.17215

Google Scholar: Author Only Title Only Author and Title

Liu Q, Wang C, Jiao X, Zhang H, Song L, Li Y, Gao C, Wang K (2019) Hi-TOM: a platform for high-throughput tracking of mutations induced by CRISPR/Cas systems. Sci China Life Sci 62:1-7

Google Scholar: Author Only Title Only Author and Title

Marsh JF, Rakocevic A, Mitra RM, Brocard L, Sun J, Eschstruth A, Long SR, Schultze M, Ratet P, Oldroyd GE (2007) Medicago truncatula NIN is essential for rhizobial-independent nodule organogenesis induced by autoactive calcium/calmodulin-dependent protein kinase. Plant Physiol 144: 324-35

Google Scholar: Author Only Title Only Author and Title

Murray JD (2011) Invasion by invitation: rhizobial infection in legumes. Mol Plant Microbe Interact 24: 631-639

Google Scholar: Author Only Title Only Author and Title

Oldroyd GE, Murray J, Poole P, Downie JA(2011) The rules of engagement in the legume-rhizobial symbiosis. Annual review of Genetics. 45:119-44

Google Scholar: Author Only Title Only Author and Title

Qiu L, Lin JS, Xu J, Sato S, Parniske M, Wang TL, Downie JA Xie F (2015) SCARN a novel class of SCAR protein that is required for root-hair infection during legume nodulation. PLoS Genet 11: e1005623

Google Scholar: Author Only Title Only Author and Title

Schauser L, Roussis A, Stiller J, Stougaard J (1999) A plant regulator controlling development of symbiotic root nodules. Nature 402: 191-5

Google Scholar: Author Only Title Only Author and Title

Schmutz J, Cannon SB, Schlueter J, Ma J, Mitros T, Nelson W, Hyten DL, Song Q, Thelen JJ, Cheng J, Xu D, Hellsten U (2010) Genome sequence of the palaeopolyploid soybean. Nature 463: 178-83

Google Scholar: Author Only Title Only Author and Title

Singh S, Katzer K, Lambert J, Cerri M, Parniske M (2014) CYCLOPS, a DNA-binding transcriptional activator, orchestrates symbiotic root nodule development. Cell Host Microbe 15: 139-52

Google Scholar: Author Only Title Only Author and Title

Soyano T, Hayashi M (2014) Transcriptional networks leading to symbiotic nodule organogenesis. Curr Opin Plant Biol 20: $146-54$. Google Scholar: Author Only Title Only Author and Title

Soyano T, Hirakawa H, Sato S, Hayashi M, Kawaguchi M (2014) Nodule Inception creates a long-distance negative feedback loop involved in homeostatic regulation of nodule organ production. Proc Natl Acad Sci U S A 111: 14607-12

Google Scholar: Author Only Title Only Author and Title

Soyano T, Kouchi H, Hirota A Hayashi M (2013) Nodule Inception directly targets NF-Y subunit genes to regulate essential processes of root nodule development in Lotus japonicus. PLoS Genet 9: e1003352

Google Scholar: Author Only Title Only Author and Title

Soyano T, Shimoda Y, Hayashi M (2015) NODULE INCEPTION antagonistically regulates gene expression with nitrate in Lotus japonicus. Plant Cell Physiol 56: 368-76

Google Scholar: Author Only Title Only Author and Title 
Soyano T, Shimoda Y, Kawaguchi M, Hayashi M (2019) A shared gene drives lateral root development and root nodule symbiosis pathways in Lotus. Science 366: 1021-1023

Google Scholar: Author Only Title Only Author and Title

Vaddepalli P, Fulton L, Schneitz K (2019) Asymmetric redundancy of ZERZAUST and ZERZAUST HOMOLOG in different accessions of Arabidopsis thaliana. G3 Genes|Genomics|Genetics 9: 2245-2252

Google Scholar: Author Only Title Only Author and Title

van Velzen R, Holmer R, Bu F, Rutten L, van Zeijl A, Liu W, Santuari L, Cao Q, Sharma T, Shen D, Roswanjaya Y, Wardhani TAK (2018) Comparative genomics of the nonlegume Parasponia reveals insights into evolution of nitrogen-fixing rhizobium symbioses. Proc Natl Acad Sci U S A 115: E4700-e4709

Google Scholar: Author Only Title Only Author and Title

Vernie T, Kim J, Frances L, Ding Y, Sun J, Guan D, Niebel A, Gifford ML, de Carvalho-Niebel F, Oldroyd GE (2015) The NIN transcription factor coordinates diverse nodulation programs in different tissues of the Medicago truncatula root. Plant Cell 27: 341024

Google Scholar: Author Only Title Only Author and Title

Xie F, Murray JD, Kim J, Heckmann AB, Edwards A, Oldroyd GE, Downie JA(2012) Legume pectate lyase required for root infection by rhizobia. Proc Natl Acad Sci U S A 109: 633-8

Google Scholar: Author Only Title Only Author and Title 